\title{
Hypomethylation of Agtrap is associated with long-term inhibition of left ventricular hypertrophy in prehypertensive losartan-treated spontaneously hypertensive rats
}

\author{
TING-JUN WANG, GUI-LI LIAN, XU LIN, HONG-BIN ZHONG, \\ CHANG-SHENG XU, HUA-JUN WANG and LIANG-DI XIE \\ Fujian Hypertension Research Institute, The First Clinical College of Fujian \\ Medical University, Fuzhou, Fujian 350005, P.R. China
}

Received November 15, 2015; Accepted November 10, 2016

DOI: $10.3892 / \mathrm{mmr} .2016 .6040$

\begin{abstract}
Prehypertensive losartan treatment may lead to long-term inhibition of the development of left ventricular hypertrophy $(\mathrm{LVH})$ in spontaneously hypertensive rats (SHRs). However, the underlying mechanism has yet to be fully elucidated. The aim of the present study was to investigate the expression of angiotensin type 1 receptor-associated protein (ATRAP/Agtrap) and methylation of the Agtrap gene in the myocardium following the withdrawal of treatment. Four-week-old SHRs were randomly divided into three groups, and were treated with saline, amlodipine or losartan, respectively, for 6 weeks. Wistar Kyoto rats (WKYs) were used as a control. All rats were followed up regularly until they reached the age of 32 weeks. Systolic blood pressure (SBP), left ventricular mass/body weight (LVM/BW), and cardiac fibrosis and structure were measured. The mRNA and protein expression of ATRAP in the myocardium were determined using reverse transcription-quantitative polymerase chain reaction and western blot analysis. Methylation of the Agtrap promoter was detected by bisulfite pyrosequencing. Reduced levels of SBP, LVM/BW, cardiac fibrosis and interventricular septum thickness were determined to be maintained only in prehypertensive losartan-treated SHRs. Whereas, an increased expression of ATRAP mRNA and protein, and hypomethylation of the Agtrap promoter in the myocardium, were demonstrated only in the losartan-treated SHRs. In conclusion, the results of the present study suggested that the hypomethylation of Agtrap accompanying upregulation of ATRAP expression in the myocardium is associated with the
\end{abstract}

Correspondence to: Dr Liang-Di Xie, Fujian Hypertension Research Institute, The First Clinical College of Fujian Medical University, 20 Chazhong Road, Fuzhou, Fujian 350005, P.R. China E-mail: xieliangdi@126.com

Key words: angiotensin type 1 receptor-associated protein, methylation, losartan, left ventricular hypertrophy, prehypertension, spontaneously hypertensive rats long-term inhibition of LVH in SHRs with prehypertensive losartan treatment.

\section{Introduction}

Hypertension-associated left ventricular hypertrophy (LVH) is considered to be an independent risk factor of adverse cardiovascular events, including arrhythmia, myocardial infarction, congestive heart failure, stroke and kidney failure (1-6). In spontaneously hypertensive rats (SHRs), prehypertensive treatment with losartan, a renin-angiotensin system (RAS) inhibitor, may prevent the development of LVH in the long term. By contrast, the calcium-channel blocker, amlodipine, another class of antihypertensive drug, fails to maintain the beneficial effects following the withdrawal of treatment. Beyond prolongation of the blood pressure-lowering effect, a decrease in the levels of certain RAS components in the myocardium, including angiotensin II (Ang II) and Ang II type 1 receptor (AT1R), may explain the inhibition of $\mathrm{LVH}$ induced by early losartan treatment (7). It is well established that RAS comprises a number of components, and the various components collaborate to regulate homeostasis and pathogenesis of the cardiovascular system (8). Therefore, it is reasonable to hypothesize that changes occur in the other components of RAS in the myocardium of losartan-treated SHRs.

AT1R-associated protein (ATRAP/Agtrap), a newly identified component of RAS, specifically binds to AT1R, promotes internalization of the receptor, and downregulates the AT1R-mediated signaling pathway (9). Overexpression of ATRAP attenuates cardiomyocyte hypertrophy in vitro (10), reduces oxidative stress and inflammation in local tissue in vivo $(11,12)$, and inhibits $\mathrm{LVH}$ in chronic Ang II-infused mice (13). Furthermore, an imbalance of AT1R and ATRAP in the myocardium is associated with the development of hypertension-associated LVH (14). However, there are few previously published studies on ATRAP expression in the myocardium of prehypertensive treated SHRs (15).

In the present study, prehypertensive SHRs were treated with losartan or amlodipine. In addition to LVM/BW, cardiac fibrosis and structure, the expression of ATRAP was observed in the myocardium of adult rats. It is well known that the 
expression of genes may be regulated by genomic variation or epigenetic modification. The latter includes DNA methylation. Therefore, an aim of the present study was to measure methylation of the Agtrap promoter in the myocardium of treated SHRs.

\section{Materials and methods}

Animals and pharmacological treatment. All animal procedures were approved by the Institutional Animal Care Committee of the First Affiliated Hospital of Fujian Medical University, China (approval no. 001). Rats were purchased from Vital River Laboratory Animal Technology (Beijing, China), and housed (5 rats/cage) at a constant ambient temperature of $22-24^{\circ} \mathrm{C}$ and a humidity of $40-60 \%$, and were exposed to a 12-h light/dark cycle. All the rats were fed standard rat chow and tap water ad libitum. A total of 724 -week-old male SHRs were randomly divided into three groups, and were administered saline ( $2 \mathrm{ml} / \mathrm{kg}$ per day; the SHR group; $\mathrm{n}=24)$, amlodipine (Aml) $[10 \mathrm{mg} / \mathrm{kg} / \mathrm{day}$; purchased from Pfizer, Inc. (New York, NY, USA); the SHR-Aml group; $n=24]$, losartan [20 mg/kg per day; purchased from Merck Sharp \& Dohme (Hangzhou, China); the SHR-Los group; $\mathrm{n}=24$ ] by gavage for 6 weeks. Wistar Kyoto rats (WKYs), treated with an equal volume of saline, were used as the control group (WKY; $n=24$ ). At 14, 20 and 32 weeks of age, eight rats in each group were anesthetized with chloral hydrate $(300 \mathrm{mg} / 100 \mathrm{~g}$, administered intraperitoneally), and underwent echocardiography prior to sacrifice.

Measurement of blood pressure and evaluation of LVH. Systolic blood pressure (SBP) of the conscious rats was measured using the tail-cuff method (Softron BP-98A; Softron $^{\mathrm{TM}}$, Beijing, China) as previously described (16), and was recorded as the mean of three consecutive readings. Body weight (BW) was measured using a balance prior to sacrifice. The chests of the rats were opened, their hearts were removed and blotted dry following sacrifice. The left ventricular mass (LVM), including the ventricular septum, was weighed, and LVM/BW was calculated to determine cardiac hypertrophy.

Determination of cardiac fibrosis. Cardiac fibrosis was measured as previously described in our laboratory (17). Briefly, the hearts were immersed into $4 \%$ formalin for $24 \mathrm{~h}$, embedded in paraffin and cut into $4-\mu \mathrm{m}$ sections for Sirius Red staining. Sections were observed under a light microscope (CX31; Olympus Corp., Tokyo, Japan). Images were analyzed using Image Pro Plus 6.0 software (Media Cybernetics. Inc., Rockville, MD, USA). The collagen volume fraction (CVF) in the ventricular septum, an index of cardiac fibrosis, was determined as the percentage of the Sirius Red-stained area/total ventricular septum area.

Echocardiography for cardiac structure and function. Rats were anesthetized as described above, and transthoracic echocardiography was subsequently performed in the left lateral decubitus position. An M-mode echocardiogram was obtained from the long-axis view of the left ventricle (LV) using a vivid 7 echocardiographic system (GE Healthcare, Piscataway, NJ, USA) with a $10 \mathrm{MHz}$ probe. The left ventricular end-diastolic dimension (LVEDD), end-diastolic interventricular septum thickness (IVSTd) and left ventricular ejection fraction (LVEF) were measured according to the guidelines of the American Society of Echocardiography (18).

Real-time quantitative reverse transcription polymerase chain reaction ( $R T-q P C R)$ for ATRAP $m R N A$. Total RNA from the LV tissue was extracted with TRIzol reagent (Invitrogen; Thermo Fisher Scientific, Inc., Waltham, MA, USA) according to the manufacturer's protocol. Total RNA $(2 \mu \mathrm{g})$ was reverse-transcribed into complementary DNA (cDNA) using a RevertAid First Strand cDNA synthesis kit (Thermo Fisher Scientific, Inc.). RT-qPCR was performed using SYBR Green PCR Master mix (Takara Bio, Inc., Otsu, Japan) and detected using an ABI Prism7000 sequence detection system (Applied Biosystems Life Technologies, Foster City, CA, USA). Thermocycler conditions consisted of an initial activation step at $95^{\circ} \mathrm{C}(30 \mathrm{~min})$, followed by a three-step PCR program of $95^{\circ} \mathrm{C}$ (for $10 \mathrm{sec}$ ), $60^{\circ} \mathrm{C}$ (for $31 \mathrm{sec}$ ) and $72^{\circ} \mathrm{C}$ $(30 \mathrm{sec})$ for 40 cycles for primer denaturation, annealing and extension, respectively. The mRNA level was quantified by calculating the values of the $\Delta$ cycle threshold $\left(\Delta \mathrm{C}_{\mathrm{q}}\right)$ by normalizing the average $\mathrm{C}_{\mathrm{q}}$ value compared with its internal control (Gapdh), and then calculating $2^{-\Delta \Delta C q}$ (19). The primer sequences were as follows: Agtrap: 5'-ACTCTGTTGATG CCATTG-3' (forward); 5'-GAAGATGTAATGTAGATA ATGTC-3' (reverse); Gapdh: 5'-CCTGCACCACCAACT GCTTA-3' (forward), 5'-AGT GATGGCATGGACTGTGG-3' (reverse).

Western blot analysis for the expression of ATRAP protein. Western blotting was performed as previously described in our laboratory, although with minor modifications (20). Briefly, the total protein in LV was extracted, and the concentrations were determined using a bicinchoninic acid protein concentration determination kit (Beyotime Institute of Biotechnology, Beijing, China). Aliquots of $80 \mu \mathrm{g}$ protein lysates extracted from the hearts were separated using 10\% SDS-PAGE gels, electro-transferred onto polyvinylidene fluoride membranes, blocked with 5\% non-fat milk in TBS containing $0.05 \%$ Tween-20 (TBST), and the protein was probed with anti-ATRAP antibody (1:200; cat. no. sc134652; Santa Cruz Biotechnology, Inc., Santa Cruz, CA, USA) or anti- $\beta$-actin antibody (1:1,000; cat. no. sc1616; Santa Cruz Biotechnology, Inc) overnight at $4^{\circ} \mathrm{C}$. After 3 washes with TBST for $5 \mathrm{~min}$, the membranes were incubated for $1 \mathrm{~h}$ at room temperature with horseradish peroxidase-conjugated secondary antibody (1:5,000; cat. nos. ZB-2301; ZB-2305; OriGene Technologies, Inc., Beijing, China). Following another 3 washes with TBST for $10 \mathrm{~min}$, protein bands were visualized using electrochemiluminescence on high-performance chemiluminescence film (Lulong Biotech Co., Ltd., Xiamen, China), and were quantified using Image Pro Plus version 6.0 (Media Cybernetics, Inc., Rockville, MD, USA).

CpG island analysis of the Agtrap promoter region. $\mathrm{CpG}$ islands in the Agtrap promoter region were analyzed using the online tool, Methprimer (http://www.urogene. org/methprimer/index.html) with parameters window100, shift1, observed $\mathrm{CpG} /$ expected $\mathrm{CpG} \geq 0.60$ and $\mathrm{GC} \% \geq 50.0 \%$. 


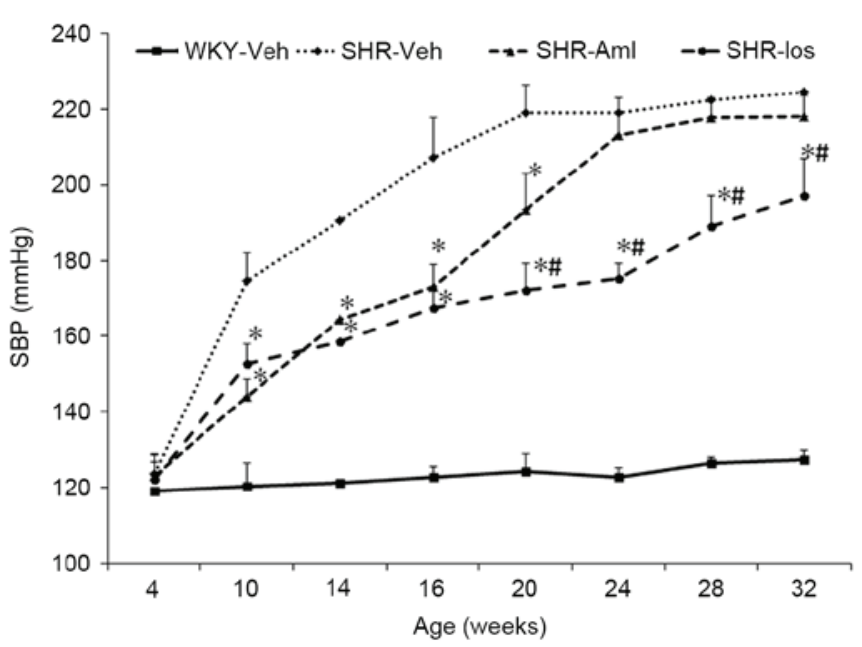

Figure 1. Long-term effect of prehypertensive losartan and amlodipine treatment on SBP in SHRs. "P $<0.05$ vs. age-matched SHR; ${ }^{\#} \mathrm{P}<0.05$ vs. age-matched SHR-Aml. The data are shown as the means \pm standard deviation $(n=8)$. WKY, vehicle-treated Wistar Kyoto rats; SHRs, spontaneously hypertensive rats; SHR, vehicle-treated SHRs; SHR-Aml, amlodipine-treated SHRs; SHR-Los, losartan-treated SHRs; SBP, systolic blood pressure.

Bisulfite-specific polymerase chain reaction (BSP-PCR) and $D N A$ sequencing for methylation of the Agtrap promoter. Genomic DNA in LV was extracted using a GenElute Mammalian Genomic DNA mini-Prep kit (Sigma-Aldrich; Merck Millipore, Darmstadt, Germany) following the manufacturer's protocol, and was subsequently subjected to treatment with the EpiTect Bisulfite Kit (Qiagen, Hilden, Germany). The bisulfite-modified DNA was amplified by PCR using BSP-specific primer pairs [GeneTech (Shanghai) Co., Ltd., Shanghai, China] under the following conditions: $95^{\circ} \mathrm{C}$ for $3 \mathrm{~min}$; followed by 40 cycles of $95^{\circ} \mathrm{C}$ for $15 \mathrm{sec}, 54^{\circ} \mathrm{C}$ for $30 \mathrm{sec}$, $72^{\circ} \mathrm{C}$ for $30 \mathrm{sec}$; and $72^{\circ} \mathrm{C}$ for $5 \mathrm{~min}$. The PCR products were sequenced using a PyroMark ID sequencer (Biotage $\mathrm{AB}$, Uppsala, Sweden) according to the manufacturer's protocol. The percentage of methylated $\mathrm{CpG}$ at each site was quantified using the Pyro Q-CpG software, version 1.0.9 (Biotage AB). The primers for BSP and DNA sequencing were as follows: Agtrap: 5'-TGGTAGAGGTTTAGGTAGTAGTAGGAGT-3' (forward); 5'-biotin-CCAACTCCAAAACAAACTTCCT-3'. (reverse); and 5'-GTTTTGTAGTAAGGGTAAT-3' (sequencing).

Statistical analysis. Statistical analysis was performed using SPSS version, 17.0 (SPSS, Inc., Chicago, IL, USA). Continuous data are presented as the means \pm standard deviation. Differences between groups were compared by one-way analysis of variance, followed by a least significant difference t-test for multiple comparisons. $\mathrm{P}<0.05$ was considered to indicate a statistically significant difference.

\section{Results}

Prehypertensive losartan treatment reduces $S B P$ in adult SHRs. SBP in the SHR group increased with age, to a maximum for the rats at 20 weeks of age, and this higher level was maintained until the rats were 32 weeks old. However, there was no change of SBP with age in the WKY group. In addition, SBP was lower in the SHR-Aml and SHR-Los
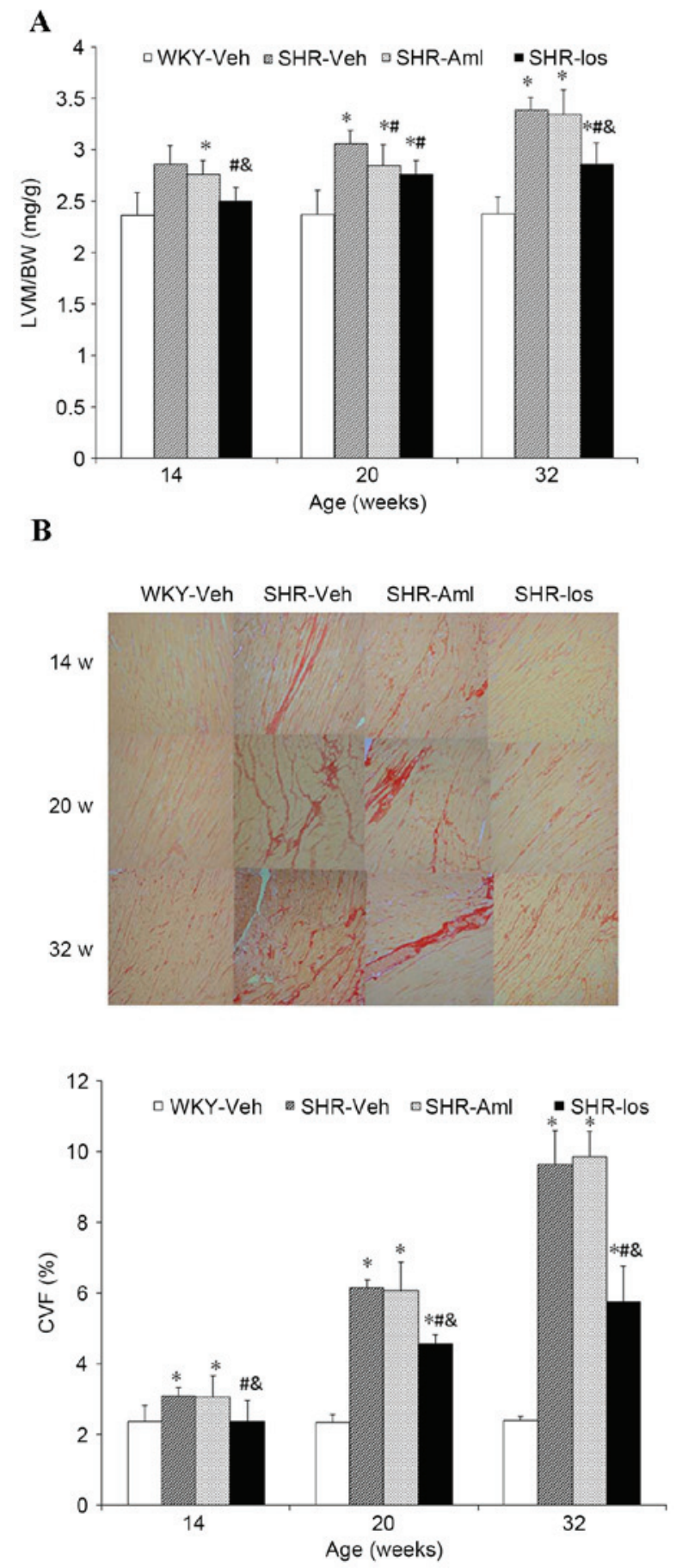

Figure 2. Long-term effect of prehypertensive losartan and amlodipine treatment on LVM/BW and CVF in the myocardium of SHRs. (A) LVM/BW at the different ages. (B) Representative pictures of myocardial collagen in ventricular septum at different ages are shown (original magnification, x10), and CVF at the different ages. The sections of ventricular septum were stained with Sirius Red. Myocardial interstitial collagen appears in red, and cardiac myocytes in yellow. " $\mathrm{P}<0.05$ vs. age-matched WKY; ${ }^{\#} \mathrm{P}<0.05$ vs. age-matched SHR; ${ }^{\&} \mathrm{P}<0.05$ vs. age-matched SHR-Aml. The data are represented as the means \pm standard deviation $(n=8)$. WKY, vehicle-treated Wistar Kyoto rats; SHRs, spontaneously hypertensive rats; SHR, vehicle-treated SHRs; SHR-Aml, amlodipine-treated SHRs; SHR-Los, losartan-treated SHRs; LVM/BW, left ventricle mass/body weight; CVF, collagen volume fraction.

groups compared with SHR before the rats reached the age of 20 weeks (for the 20-week-old rats in the three groups: SHR-Aml, $193 \pm 16 \mathrm{mmHg}$ and SHR-Los, $172 \pm 14 \mathrm{mmHg}$ vs. SHR, $219 \pm 14 \mathrm{mmHg} ; \mathrm{P}<0.001)$. From the age of 24 weeks 
Table I. Echocardiographic evaluation of the long-term effect of prehypertensive losartan and amlodipine treatment on left ventricle structure and function in spontaneously hypertensive rats.

\begin{tabular}{lcccc}
\hline Group & Age (weeks) & IVSTd (mm) & LVEDD (mm) & EF (\%) \\
\hline WKY & 10 & $1.54 \pm 0.10$ & $7.0 \pm 0.6$ & $84.2 \pm 2.1$ \\
& 20 & $1.76 \pm 0.17$ & $7.3 \pm 0.7$ & $84.3 \pm 2.6$ \\
\multirow{2}{*}{ SHR } & 32 & $1.81 \pm 0.20$ & $7.3 \pm 0.4$ & $83.2 \pm 1.9$ \\
& 10 & $2.32 \pm 0.19^{\mathrm{a}}$ & $6.9 \pm 0.8$ & $81.2 \pm 3.1$ \\
SHR-Aml & 20 & $2.54 \pm 0.17^{\mathrm{a}}$ & $6.9 \pm 0.6$ & $80.7 \pm 2.6$ \\
& 32 & $2.56 \pm 0.19^{\mathrm{a}}$ & $7.1 \pm 0.9$ & $82.7 \pm 2.9$ \\
& 10 & $2.30 \pm 0.31^{\mathrm{a}}$ & $6.9 \pm 0.9$ & $82.2 \pm 3.1$ \\
SHR-Los & 20 & $2.45 \pm 0.38^{\mathrm{a}}$ & $7.1 \pm 0.9$ & $89.9 \pm 4.6$ \\
& 32 & $2.55 \pm 0.27^{\mathrm{a}}$ & $7.4 \pm 0.5$ & $82.7 \pm 2.9$ \\
& 10 & $1.76 \pm 0.27^{\mathrm{a}, \mathrm{b}, \mathrm{c}}$ & $7.2 \pm 0.6$ & $83.2 \pm 4.1$ \\
& 20 & $1.81 \pm 0.22^{\mathrm{b}, \mathrm{c}}$ & $7.3 \pm 0.9$ & $82.9 \pm 4.6$ \\
& 32 & $1.84 \pm 0.24^{\mathrm{b}, \mathrm{c}}$ & $7.3 \pm 0.8$ & $82.7 \pm 5.0$ \\
\hline
\end{tabular}

${ }^{\mathrm{a} P}<0.05$ vs. age-matched WKY, ${ }^{\mathrm{b}}<0.05$ vs. age-matched $\mathrm{SHR},{ }^{\mathrm{C}} \mathrm{P}<0.05$ vs. age-matched SHR-Aml. WKY, vehicle-treated Wistar Kyoto rats; SHR, vehicle-treated spontaneously hypertensive rats (SHRs); SHR-Aml, amlodipine-treated SHRs; SHR-Los, losartan-treated SHRs; IVSTd, end-diastolic interventricular septum thickness; LVEDD, left ventricular end-diastolic dimension; EF, ejection fraction. The data are represented as the means \pm standard deviation $(n=8)$.

onwards, SBP in the SHR-Aml group increased to the level of the SHR group (32 weeks old: SHR-Aml, $218 \pm 12 \mathrm{mmHg}$ vs. SHR, $224 \pm 7 \mathrm{mmHg} ; \mathrm{P}=0.281$ ); however, the rats treated with losartan had a significantly lower SBP until they reached 32 weeks of age (32 weeks old: SHR-Los, $197 \pm 17 \mathrm{mmHg}$ vs. SHR, 224 \pm 7 mmHg; P<0.001) (Fig. 1).

Prehypertensive losartan treatment inhibits $\mathrm{LVH}$ and cardiac fibrosis. LVM/BW (Fig. 2A) and CVF (Fig. 2B) in the SHR group were significantly higher compared with the age-matched WKY group (LVM/BW at 32 weeks of age: SHR $3.38 \pm 0.12 \mathrm{mg} / \mathrm{g}$ vs. WKY $2.38 \pm 0.16 \mathrm{mg} / \mathrm{g}, \mathrm{P}<0.001 ; \mathrm{CVF}$, SHR $9.64 \pm 0.95 \%$ vs. WKY $2.39 \pm 0.12 \%, \mathrm{P}<0.001)$, and a marked decrease was observed in SHR-Los compared with the SHR group throughout the study period (LVM/BW at 32 weeks of age: SHR-Los, $2.86 \pm 0.21 \mathrm{mg} / \mathrm{g}$ vs. SHR, $3.38 \pm 0.12 \mathrm{mg} / \mathrm{g}$, $\mathrm{P}<0.001$; CVF, SHR-Los $5.76 \pm 0.99 \%$ vs. SHR $9.64 \pm 0.95 \%$, $\mathrm{P}<0.001$ ) (Fig. $2 \mathrm{~A}$ and $\mathrm{B}$ ). LVM/BW in the SHR-Aml group was lower than SHR at 20 weeks of age (SHR-Aml $2.85 \pm 0.20 \mathrm{mg} / \mathrm{g}$ vs. SHR $3.06 \pm 0.13 \mathrm{mg} / \mathrm{g} \mathrm{P}=0.024$ ); however, there was no difference between the two groups at 14 and 32 weeks of age ( 32 weeks old: SHR-Aml $3.35 \pm 0.23 \mathrm{mg} / \mathrm{g}$ vs. SHR $3.38 \pm 0.12 \mathrm{mg} / \mathrm{g}: \mathrm{P}=0.690$ ) (Fig. $2 \mathrm{~A}$ ). $\mathrm{CVF}$ in the SHR-Aml group was comparable with that of the SHR group at the same ages throughout the study period (32 weeks old: $\mathrm{P}=0.590)$ (Fig. 2B).

The effect of prehypertensive losartan treatment on cardiac structure and function. The data obtained are shown in Table I. From 10 to 32 weeks old, IVSTd in the SHR group was greater than in WKY (32 weeks old: SHR $2.56 \pm 0.19 \mathrm{~mm}$ vs. WKY $1.81 \pm 0.20 \mathrm{~mm} \mathrm{P}<0.001)$, and comparable with SHR-Aml (32 weeks old: SHR $2.56 \pm 0.19 \mathrm{~mm}$ vs. SHR-Aml $2.55 \pm 0.27 \mathrm{~mm}, \mathrm{P}=0.939$ ); however, IVSTd was decreased

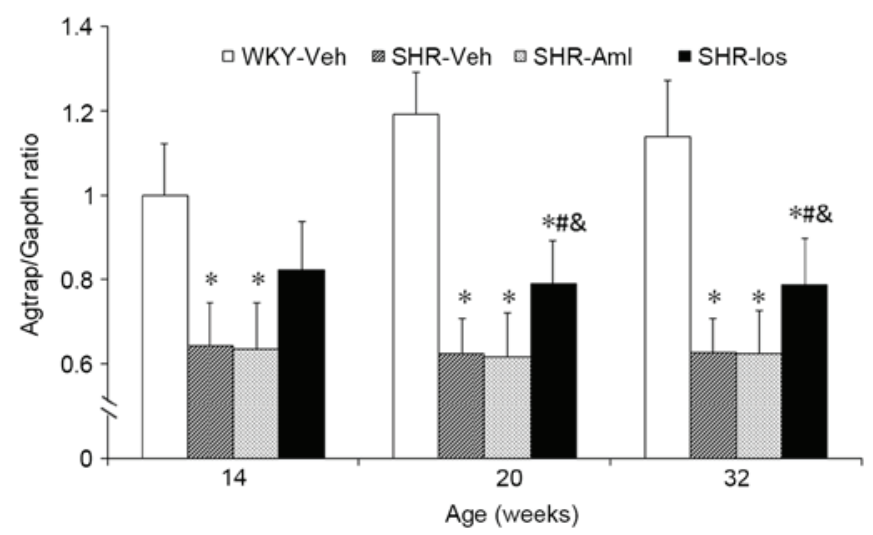

Figure 3. Long-term effect of prehypertensive losartan and amlodipine treatment on the mRNA expression of ATRAP in the myocardium of SHRs. ${ }^{*} \mathrm{P}<0.05$ vs. age-matched WKY; ${ }^{\circ} \mathrm{P}<0.05$ vs. age-matched SHR; ${ }^{\circledR} \mathrm{P}<0.05$ vs. age-matched SHR-Aml. The data are shown as the means \pm standard deviation $(\mathrm{n}=8)$. WKY, vehicle-treated Wistar Kyoto rats; SHRs, spontaneously hypertensive rats; SHR, vehicle-treated SHRs; SHR-Aml, amlodipine-treated SHRs; SHR-Los, losartan-treated SHRs; ATRAP, angiotensin II type 1 receptor-associated protein.

in the SHR-Los group compared with SHR (32 weeks old: SHR-Los $1.84 \pm 0.24 \mathrm{~mm}$ vs. SHR $2.56 \pm 0.19 \mathrm{~mm}, \mathrm{P}<0.001$ ). No significant difference was observed in LVEDD and LVEF among the four groups of age-matched rats throughout the study period (LVEDD at 32 weeks old: $\mathrm{P}=0.851 ; \mathrm{EF}: \mathrm{P}=0.988$ ).

Prehypertensive losartan treatment upregulates the levels of ATRAP mRNA and protein in the myocardium. The mRNA level of ATRAP in the SHR group was decreased compared with age-matched WKY throughout the study period (32 weeks old: SHR $0.63 \pm 0.08$ vs. WKY $1.18 \pm 0.17, \mathrm{P}<0.001$ ). The expression level of mRNA in the SHR-Los group was 
$\mathbf{A}$
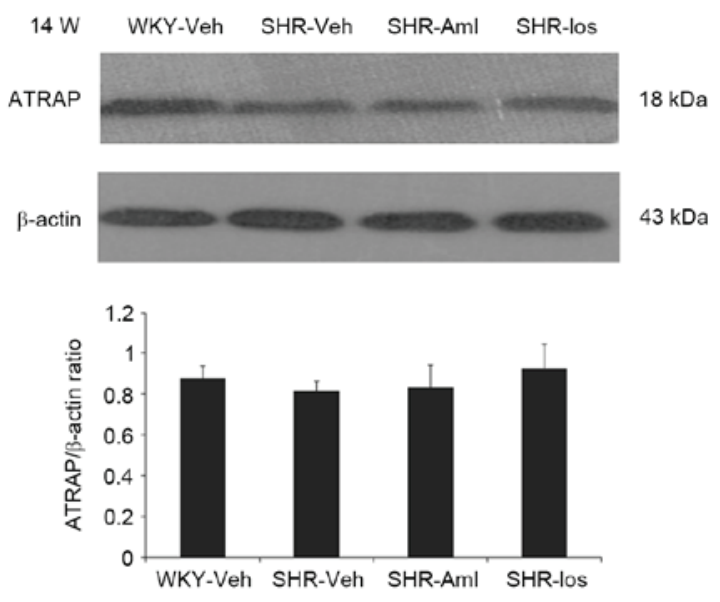

B

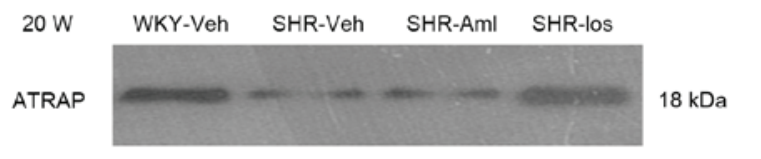

$\beta$-actin
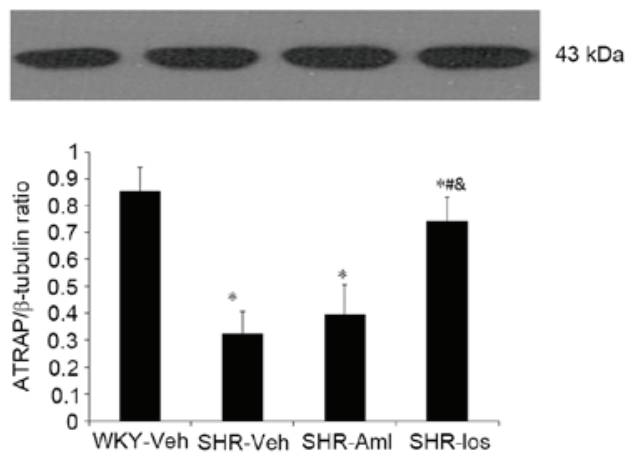

C
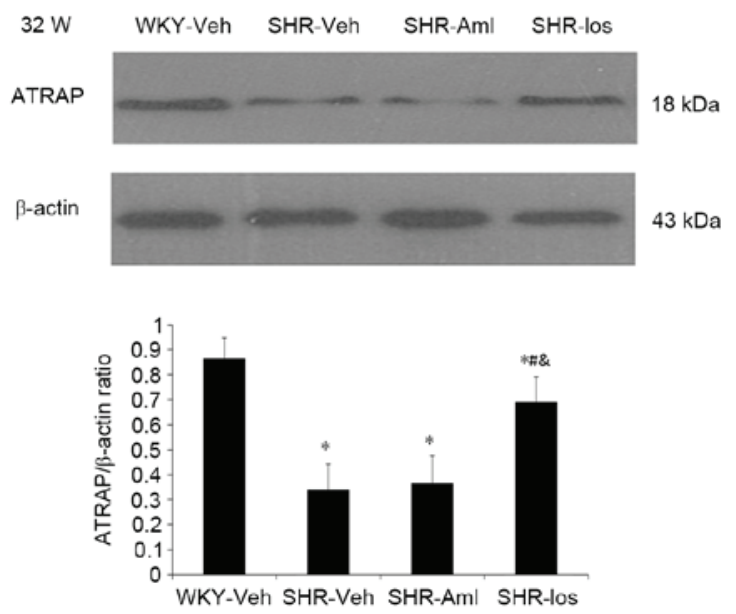

Figure 4. Long-term effect of prehypertensive losartan and amlodipine treatment on the protein expression of ATRAP in the myocardium of SHRs. The protein expression of ATRAP was determined (A) 14, (B) 20 and (C) 32 weeks following treatment. ${ }^{\mathrm{P}}<0.05$ vs. age-matched WKY; ${ }^{\mathrm{P}}<0.05$ vs. age-matched SHR; ${ }^{\&} \mathrm{P}<0.05$ vs. age-matched SHR-Aml. The data are shown as the means \pm standard deviation $(n=8)$. WKY, vehicle-treated Wistar Kyoto rats; SHRs, spontaneously hypertensive rats; SHR, vehicle-treated SHRs; SHR-Aml, amlodipine-treated SHRs; SHR-Los, losartan-treated SHRs; ATRAP, angiotensin II type 1 receptor-associated protein.

elevated compared with SHR (at 32 weeks of age: SHR-Los, $0.79 \pm 0.11$ vs. SHR, 0.63 $\pm 0.08 ; \mathrm{P}=0.014$ ) (Fig. 3). As shown in Fig. 4, no significant differences were observed in the protein level of ATRAP at 14 weeks among the four groups of rats $(\mathrm{P}=0.057)$. However, at the ages of 20 weeks and 32 weeks, the protein level in the SHR group was decreased compared with age-matched WKY (at 32 weeks of age: SHR $0.34 \pm 0.10$ vs. WKY 1.04 $\pm 0.10, \mathrm{P}<0.001$ ), and losartan-treated SHRs demonstrated an increased protein level compared with the SHR group (at 32 weeks of age: SHR-Los, $0.69 \pm 0.10$ vs. SHR0.34 \pm 0.10 ; $\mathrm{P}<0.001$. No significant differences were observed in the expression levels of ATRAP mRNA and protein between the SHR and age-matched SHR-Aml groups throughout the study period (mRNA at 32 weeks of age: $\mathrm{P}=0.883$; protein, $\mathrm{P}=0.638$ ).

Prehypertensive losartan treatment decreases the methylation of Agtrap promoter in the myocardium. One $\mathrm{CpG}$ island was identified in the vicinity of the transcription start site (TSS) of Agtrap between the upstream -132 bp and downstream +144 bp (Fig. 5A). As shown in Fig. 5B, changes in the methylation level among the four groups of the rats were observed at the $-120,-117$, -85 and -65 bp sites in the $\mathrm{CpG}$ islands, but not at the other $\mathrm{CpG}$ sites (Fig. 5B). The methylation level at the -120 bp site was higher in the SHR group than in WKY throughout the study period (at 32 weeks old: SHR $17.6 \pm 2.3 \%$ vs. WKY $4.8 \pm 0.9 \% \mathrm{P}<0.001$ ), and although no significant differences were observed between the SHR and SHR-Aml groups (at 32 weeks old: SHR 17.6 $\pm 2.3 \%$ vs. SHR-Aml $16.4 \pm 2.0 \%, \mathrm{P}=0.187$ ), the level was reduced in the age-matched SHR-Los group (at 32 weeks old: SHR 17.6 $\pm 2.3 \%$ vs. SHR-Los $6.4 \pm 1.5 \%, \mathrm{P}<0.001$ ) (Fig. 5C). Similar changes were identified at the $-117,-85$ and -65 bp sites; furthermore, the methylation levels at the three target sites in the SHR-Los group were comparable with those of the age-matched WKY group (-117, -85 and -65 bp at 32 weeks old: $\mathrm{P}=0.247$; Fig. 5D-F).

\section{Discussion}

In the present study, it has been demonstrated that prehypertensive losartan treatment was more effective than amlodipine in preventing the progression of $\mathrm{LVH}$ and cardiac fibrosis in adult SHRs. It was also shown that the beneficial effect of early losartan treatment was associated with the upregulation of ATRAP expression and hypomethylation of the Agtrap promoter in the myocardium. 
A

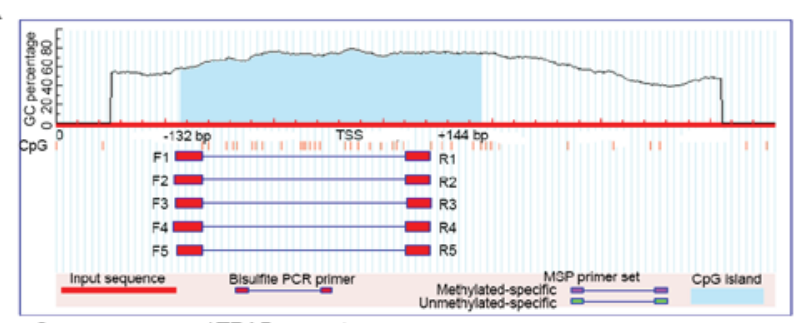

Sequence name: ATRAP promoter Sequence length: 659

CPG island prediction results

(Criteria used: island size $>100, \mathrm{GC}$ percent $>50.0, \mathrm{Obs} / \mathrm{Exp}>0.6$ )

$1 \mathrm{CpG}$ island (s) were found in your sequence

Size (Start - End)

Island 1276 bp $\quad(115-390)$

TSS:transcript start site

C

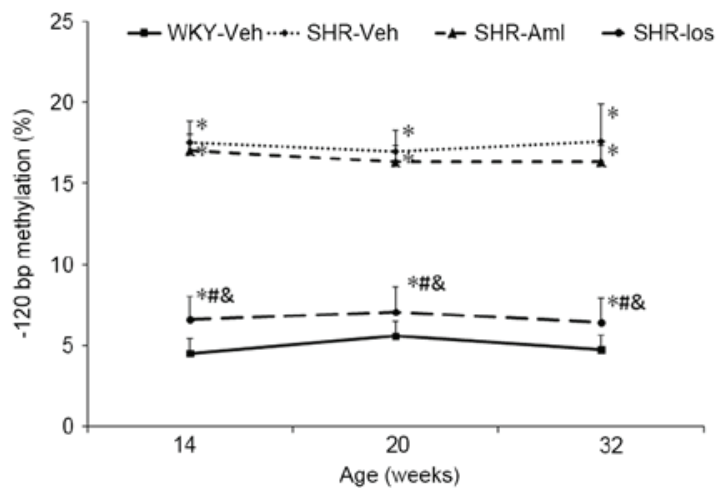

$\mathbf{E}$

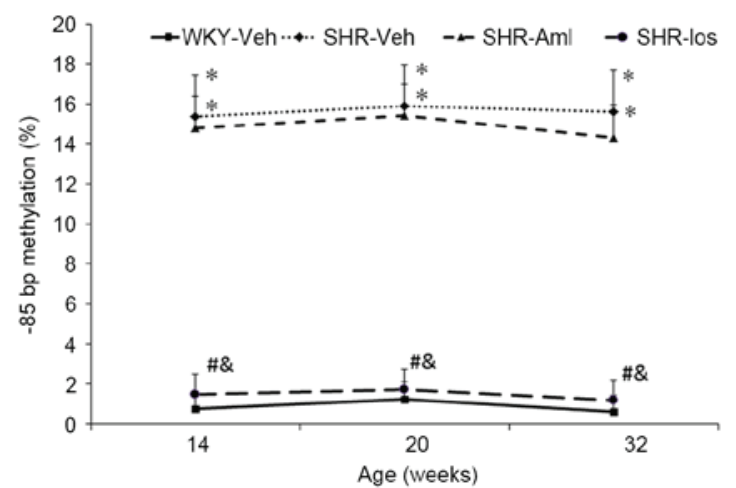

B

$-120-117 \quad-103$

agcaggagcc tgagcttgc agcaagggca agctgctaga cgecfgtctcc tagcaadgga

$$
-91-85-81-81 \quad-68-65
$$

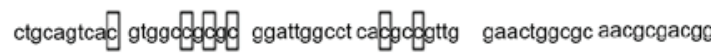

$-1$

gcggggecag gaagtttgtt cccgagttcg gagcccagga gtccccgtgg ccgtcgctca
D

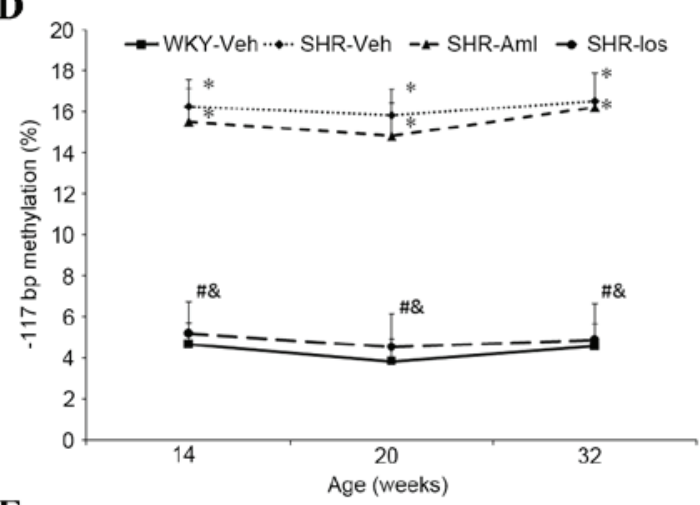

F

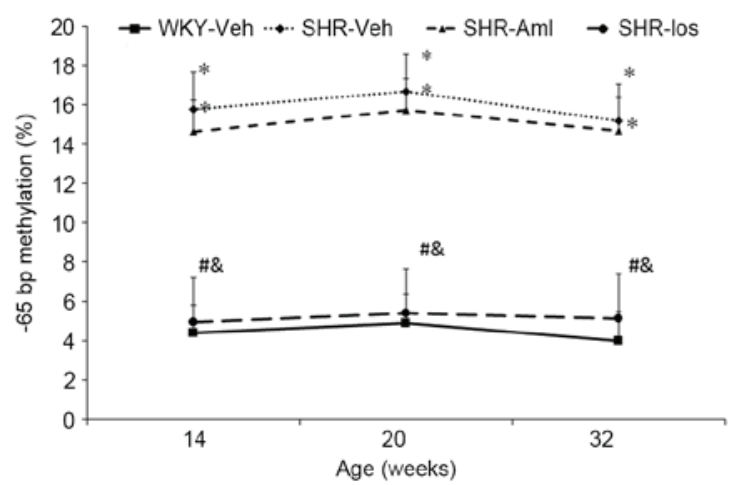

Figure 5. Long-term effects of prehypertensive losartan and amlodipine treatment on methylation of the Agtrap promoter in the myocardium of SHRs. (A) Prediction result of $\mathrm{CpG}$ islands in the Agtrap promoter: The blue zone corresponds to CpG-pattern-rich regions, whereas the red vertical lines correspond to positions of the $\mathrm{CpG}$ sites. (B) $\mathrm{CpG}$ sites in the vicinity of the Agtrap transcription start site: Red numbers represent altered CpG sites among the four groups of rats, whereas the yellow zone represents the transcript start site (C-F) Quantification of the methylation status of (C) -120 bp, (D) -117 bp, (E) - 85 bp and (F) $-65 \mathrm{bp}$ CpG sites at the different ages. The data are shown as the means \pm standard deviation $(\mathrm{n}=8)$. ${ }^{*} \mathrm{P}<0.05$ vs. age-matched WKY; ${ }^{\mathrm{P}}<0.05$ vs. age-matched SHR; ${ }^{\circledR} \mathrm{P}<0.05$ vs. age-matched SHR-Aml. WKY, vehicle-treated Wistar Kyoto rats; SHRs, spontaneously hypertensive rats; SHR, vehicle-treated SHRs; SHR-Aml, amlodipine-treated SHRs; SHR-Los, losartan-treated SHRs; Agtrap, angiotensin II type 1 receptor-associated protein gene.

Previous evidence has shown that prehypertensive patients are more likely to progress to manifest hypertension than patients with optimal or normal blood pressure. Certain prehypertensive patients may even be at an increased risk of mortality from cardiovascular disease $(21,22)$. However, it has yet to be determined whether early treatment is able to prevent the pathogenesis of hypertension. The present study has revealed that prehypertensive losartan treatment was able to reduce SBP, LVM/BW and CVF in adult SHRs. The TRial Of Preventing HYpertension ('TROPHY') and Prevention of hypertension with the angiotensin-converting enzyme inhibitor, ramipril ('PHARAO') trials demonstrated that prehypertensive treatment with RAS inhibitor over a relatively short time period reduced the risk of incident hypertension $(23,24)$. In addition, animal studies that featured early L-arginine and antioxidant supplements or captopril demonstrated a delay in the increase of blood pressure in adult SHRs (25-27). Nevertheless, the administration of the antihypertensive drug, amlodipine, or treatment with a $\beta$-blocker led to no effect on long-term blood pressure in SHRs (28). These findings suggested that, although prehypertensive treatment is an attractive alternative, the use of proper medicines remains important.

In the present study, the upregulation of ATRAP expression was revealed in losartan-treated SHRs, suggesting that ATRAP may be partly responsible for the beneficial effects elicited by early losartan treatment on LVH. It may be hypothesized 
that ATRAP inhibited LVH through several potential pathways. One possibility is that ATRAP downregulated p38 mitogen-activated protein kinase (p38-MAPK) whereas p38-MAPK could mediate negative inotropic action $(29,30)$. Secondly, ATRAP may inhibit the signal transducer and activator of transcription 3 (STAT3) signaling pathway (29). It has been reported that the STAT3 signaling pathway is associated with an increase in microtubule stabilization, and a decrease in contractility in the myocardium (31). Therefore, ATRAP may increase the contractility of cardiomyocytes. Finally, it is also possible that ATRAP blocks the calcineurin/nuclear factor of activated T cells (NFAT) pathway (32) and, consequently, gene expression associated with cardiomyocyte hypertrophy is downregulated. Taken together, ATRAP may attenuate compensatory LVH through different pathways.

LVH occurs due to the adaptation to pressure or volume overload with an overactivation of protein turnover. Protein balance is regulated by synthesis and degradation. Li et al (29) demonstrated that the level of ATRAP was controlled by proteasome degradation, whereas protein synthesis may be regulated at the translational and transcriptional levels, including via the process of methylation modification. It was predicted that one $\mathrm{CpG}$ island would be located in the vicinity of the TSS of the Agtrap promoter. Our data have further demonstrated that methylation of the Agtrap promoter was negatively correlated with the expression of ATRAP mRNA, suggesting that ATRAP expression may be regulated by methylation modification. A burgeoning body of evidence has shown that the methylation of specific genes is involved in the pathogenesis of genetic hypertension and associated target organ damage $(33,34)$. For example, Cho et al $(35)$ revealed that methylation of the $\mathrm{Na}^{+}-\mathrm{K}^{+}-2 \mathrm{Cl}^{-}$co-transporter 1 was decreased in the aorta and heart of adult SHRs, and Pei et al (36) demonstrated that hypomethylation of ATRAP in the aorta and mesenteric artery of SHRs was accompanied by increased blood pressure. Furthermore, Watson et al demonstrated that the DNA methylation inhibitor, 5-azacytidine, was able to prevent the development of LVH in SHRs (37). Therefore, these studies suggested that prehypertensive losartan treatment reduces the DNA methylation level of Agtrap, resulting in the upregulation of ATRAP expression in the myocardium, which consequently leads to an inhibition of the development of LVH in adult SHRs.

Methylation is catalyzed by DNA methyltransferases (38), and it may be triggered by oxidative stress (39). Further studies are required to better understand the mechanism of the hypomethylation of Agtrap.

In conclusion, the results presented in the current study have demonstrated that hypomethylation of Agtrap in the myocardium was associated with an increased expression of ATRAP and an attenuation of LVH in prehypertensive losartan-treated SHRs, providing a novel insight into the beneficial effects elicited by an early RAS inhibitor.

\section{Acknowledgements}

This study was supported by a grant from a key program of Fujian Medical University (no. XK201107). The authors would like to thank Li Liu for her technical assistance in editing this manuscript.

\section{References}

1. Taylor J: 2013 ESH/ESC guidelines for the management of arterial hypertension. Eur Heart J 34: 2108-2109, 2013.

2. Chatterjee S, Bavishi C, Sardar P, Agarwal V, Krishnamoorthy P, Grodzicki T and Messerli FH: Meta-analysis of left ventricular hypertrophy and sustained arrhythmias. Am J Cardiol 114: 1049-1052, 2014

3. Levy D, Garrison RJ, Savage DD, Kannel WB and Castelli WP: Prognostic implications of echocardiographically determined left ventricular mass in the Framingham Heart Study. N Engl J Med 322: 1561-1566, 1990.

4. Okin PM, Devereux RB, Nieminen MS, Jern S, Oikarinen L, Viitasalo M, Toivonen L, Kjeldsen SE, Julius S, Snapinn S, et al: Electrocardiographic strain pattern and prediction of cardiovascular morbidity and mortality in hypertensive patients Hypertension 44: 48-54, 2004.

5. Wang S, Xue H, Zou Y, Sun K, Fu C, Wang H and Hui R: Left ventricular hypertrophy, abnormal ventricular geometry and relative wall thickness are associated with increased risk of stroke in hypertensive patients among the Han Chinese. Hypertens Res 37: 870-874, 2014

6. Charytan D: Is left ventricular hypertrophy a modifiable risk factor in end-stage renal disease. Curr Opin Nephrol Hypertens 23: 578-585, 2014.

7. Peng F, Lin J, Lin L and Tang H: Transient prehypertensive treatment in spontaneously hypertensive rats: A comparison of losartan and amlodipine regarding long-term blood pressure, cardiac and renal protection. Int J Mol Med 30: 1376-1386, 2012.

8. Horiuchi M, Iwanami J and Mogi M: Regulation of angiotensin II receptors beyond the classical pathway. Clin Sci (Lond) 123: 193-203, 2012.

9. Lopez-Ilasaca M, Liu X, Tamura K and Dzau VJ: The angiotensin II type I receptor-associated protein, ATRAP, is a transmembrane protein and a modulator of angiotensin II signaling. Mol Biol Cell 14: 5038-5050, 2003.

10. Tanaka Y, Tamura K, Koide Y, Sakai M, Tsurumi Y, Noda Y, Umemura M, Ishigami $\mathrm{T}$, Uchino $\mathrm{K}$, Kimura $\mathrm{K}$, et al: The novel angiotensin II type 1 receptor (AT1R)-associated protein ATRAP downregulates AT1R and ameliorates cardiomyocyte hypertrophy. FEBS Lett 579: 1579-1586, 2005.

11. Wakui H, Dejima T, Tamura K, Uneda K, Azuma K, Maeda A, Ohsawa M, Kanaoka T, Azushima K and Kobayashi R: Activation of angiotensin II type 1 receptor-associated protein exerts an inhibitory effect on vascular hypertrophy and oxidative stress in angiotensin II-mediated hypertension. Cardiovasc Res 100: 511-519, 2013.

12. Maeda A, Tamura K, Wakui H, Dejima T, Ohsawa M, Azushima K, Kanaoka T, Uneda K, Matsuda M, Yamashita A, et al: Angiotensin receptor-binding protein ATRAP/Agtrap inhibits metabolic dysfunction with visceral obesity. J Am Heart Assoc 2: e000312, 2013.

13. Wakui H, Tamura K, Tanaka Y, Matsuda M, Bai Y, Dejima T, Masuda S, Shigenaga A, Maeda A, Mogi M, et al: Cardiac-specific activation of angiotensin II type 1 receptor-associated protein completely suppresses cardiac hypertrophy in chronic angiotensin II-infused mice. Hypertension 55: 1157-1164, 2010.

14. Shigenaga A, Tamura K, Wakui H, Masuda S, Azuma K, Tsurumi-Ikeya Y, Ozawa M, Mogi M, Matsuda M, Uchino K, et al: Effect of olmesartan on tissue expression balance between angiotensin II receptor and its inhibitory binding molecule. Hypertension 52: 672-678, 2008.

15. Baumann M, Sollinger D, Roos M, Lutz J and Heemann U: Prehypertensive preconditioning improves adult antihypertensive and cardioprotective treatment. J Pharmacol Exp Ther 332: 1121-1126, 2010.

16. Widdop RE and Li XC: A simple versatile method for measuring tail cuff systolic blood pressure in conscious rats. Clin Sci (Lond) 93: 191-194, 1997.

17. Yao J, Xie XL, Xie LD, et al: The influence of naoxintong on myocardial fibrosis in spontaneously hypertensive rats. Chin J of Integ Med 6: 188-190, 2008.

18. Lang RM, Badano LP, Mor-Avi V, Afilalo J, Armstrong A, Ernande L, Flachskampf FA, Foster E, Goldstein SA, Kuznetsova T, et al: Recommendations for cardiac chamber quantification by echocardiography in adults: An update from the American society of echocardiography and the European association of cardiovascular imaging. Eur Heart J Cardiovasc Imaging 16: 233-270, 2015. 
19. Livak KJ and Schmittgen TD: Analysis of relative gene expression data using real-time quantitative PCR and the 2(-Delta Delta C(T)) method. Methods 25: 402-408, 2001.

20. Chen HF, Xie LD and Xu CS: Role of heat shock protein 27 phosphorylation in migration of vascular smooth muscle cells Mol Cell Biochem 327: 1-6, 2009.

21. Vasan RS, Larson MG, Leip EP, Kannel WB and Levy D: Assessment of frequency of progression to hypertension in non-hypertensive participants in the Framingham Heart Study: A cohort study. Lancet 358: 1682-1686, 2001.

22. Vasan RS, Larson MG, Leip EP, Evans JC, O'Donnell CJ, Kannel WB and Levy D: Impact of high-normal blood pressure on the risk of cardiovascular disease. N Engl J Med 345: 1291-1297, 2001

23. Julius S, Nesbitt SD, Egan BM, Weber MA, Michelson EL, Kaciroti N, Black HR, Grimm RH Jr, Messerli FH, Oparil S, et al: Feasibility of treating prehypertension with an angiotensin-receptor blocker. N Engl J Med 354: 1685-1697, 2006.

24. Lüders S, Schrader J, Berger J, Unger T, Zidek W, Böhm M, Middeke M, Motz W, Lübcke C, Gansz A, et al: The PHARAO study: Prevention of hypertension with the angiotensin-converting enzyme inhibitor ramipril in patients with high-normal blood pressure: A prospective, randomized, controlled prevention trial of the German Hypertension League. J Hypertens 26: 1487-1496, 2008.

25. Racasan S, Braam B, van der Giezen DM, Goldschmeding R, Boer P, Koomans HA and Joles JA: Perinatal L-arginine and antioxidant supplements reduce adult blood pressure in spontaneously hypertensive rats. Hypertension 44: 83-88, 2004.

26. Harrap SB, Nicolaci JA and Doyle AE: Persistent effects on blood pressure and renal haemodynamics following chronic angiotensin converting enzyme inhibition with perindopril. Clin Exp Pharmacol Physiol 13: 753-765, 1986.

27. Chen DG, Jin XQ and Wang HJ: Mechanisms responsible for sustained hypotension after captopril treatment. J Hypertens 13: 1113-1121, 1995.

28. Christensen KL, Jespersen LT and Mulvany MJ: Development of blood pressure in spontaneously hypertensive rats after withdrawal of long-term treatment related to vascular structure. J Hypertens 7: 83-90, 1989.

29. Li N, Wang HX, Han QY, Li WJ, Zhang YL, Du J, Xia YL and Li HH: Activation of the cardiac proteasome promotes angiotension II-induced hypertrophy by down-regulation of ATRAP. J Mol Cell Cardiol 79: 303-314, 2015.
30. Vahebi S, Ota A, Li M, Warren CM, de Tombe PP, Wang Y and Solaro RJ: p38-MAPK induced dephosphorylation of alpha-tropomyosin is associated with depression of myocardial sarcomeric tension and ATPase activity. Circ Res 100: 408-415, 2007.

31. Ng DC, Ng IH, Yeap YY, Badrian B, Tsoutsman T, McMullen JR, Semsarian C and Bogoyevitch MA: Opposing actions of extracellular signal-regulated kinase (ERK) and signal transducer and activator of transcription 3 (STAT3) in regulating microtubule stabilization during cardiac hypertrophy. J Biol Chem 286: 1576-1587, 2011

32. Min LJ, Mogi M, Tamura K, Iwanami J, Sakata A, Fujita T, Tsukuda K, Jing F, Iwai M and Horiuchi M: Angiotensin II type 1 receptor-associated protein prevents vascular smooth muscle cell senescence via inactivation of calcineurin/nuclear factor of activated T cells pathway. J Mol Cell Cardiol 47: 798-809, 2009.

33. Xiao D, Dasgupta C, Li Y, Huang X and Zhang L: Perinatal nicotine exposure increases angiotensin II receptor-mediated vascular contractility in adult offspring. PLoS one 9: e108161, 2014.

34. Friso S, Pizzolo F, Choi SW, Guarini P, Castagna A, Ravagnani V, Carletto A, Pattini P, Corrocher R and Olivieri O: Epigenetic control of 11 beta-hydroxysteroid dehydrogenase 2 gene promoter is related to human hypertension. Atherosclerosis 199: 323-327, 2008.

35. Cho HM, Lee HA, Kim HY, Han HS and Kim IK: Expression of $\mathrm{Na}+\mathrm{K}+-2 \mathrm{Cl}^{-}$cotransporter 1 is epigenetically regulated during postnatal development of hypertension. Am J Hypertens 24: 1286-1293, 2011

36. Pei F, Wang X, Yue R, Chen C, Huang J, Huang J, Li X and Zeng C: Differential expression and DNA methylation of angiotensin type $1 \mathrm{~A}$ receptors in vascular tissues during genetic hypertension development. Mol Cell Biochem 402: 1-8, 2015

37. Watson CJ, Horgan S, Neary R, Glezeva N, Tea I, Corrigan N, McDonald K, Ledwidge M and Baugh J: Epigenetic therapy for the treatment of hypertension-induced cardiac hypertrophy and fibrosis. J Cardiovasc Pharmacol Ther 21: 127-137, 2016.

38. Subramaniam D, Thombre R, Dhar A and Anant S: DNA methyltransferases: A novel target for prevention and therapy. Front Oncol 4: 80, 2014.

39. Rexhaj E, Bloch J, Jayet PY, Rimoldi SF, Dessen P, Mathieu C, Tolsa JF, Nicod P, Scherrer U and Sartori C: Fetal programming of pulmonary vascular dysfunction in mice: Role of epigenetic mechanisms. Am J Physiol Heart Circ Physiol 301: H247-H252, 2011. 